\title{
CNS cells in cerebral malaria: active participants, not innocent bystanders Nicholas Hunt*1, Silvia Weiser ${ }^{1}$, Isabelle Medana ${ }^{2}$, Tailoi Chan-Ling 3 , Jenny Miu ${ }^{1}$, Andrew Mitchell ${ }^{1}$ and Helen Ball ${ }^{1}$
}

\begin{abstract}
Address: ${ }^{1}$ Molecular Immunopathology Unit, Bosch Institute and School of Medical Sciences, University of Sydney, Sydney, NSW 2006, Australia, ${ }^{2}$ Malaria Research Group, Nuffield Department of Clinical Laboratory Sciences, John Radcliffe Hospital, Oxford University, Oxford OX3 9DU, UK and ${ }^{3}$ Anatomy and Histology, Bosch Institute and School of Medical Sciences, University of Sydney, Sydney, NSW 2006, Australia

Email: Nicholas Hunt* - nhunt@med.usyd.edu.au

* Corresponding author
\end{abstract}

from Infectious diseases of the nervous system: pathogenesis and worldwide impact Paris, France. 10-13 September 2008

Published: 23 September 2008

BMC Proceedings 2008, 2(Suppl I):S22

This abstract is available from: http://www.biomedcentral.com/I753-656I/2/SI/S22

(c) 2008 Hunt et al; licensee BioMed Central Ltd.

Cerebral malaria is a major life-threatening complication of Plasmodium falciparum infection. A high proportion of deaths from severe malaria involves cerebral complications and occurs chiefly in children in sub-Saharan Africa. The key pathogenic mechanisms involved in cerebral malaria remain unclear. The presence of parasitized red blood cells (PRBC) within the small vessels in the central nervous system leads to derangements of brain function that result in behavioral changes, often leading to coma and death. Treatment with anti-malarial drugs can reduce mortality from cerebral malaria by around $75 \%$, but survivors frequently have neurological sequelae. Thus the pathogenesis of cerebral malaria may depend partly on acute disturbances of brain function that can be fatal, together with damage to neuronal function that is reflected in the long-term consequences in survivors. Over the last 15 years, increasing attention has been paid to the contribution of non-neuronal CNS cells to the pathogenesis of cerebral malaria, in particular the roles of endothelial cells, astrocytes and microglia.

In many cases, observations using a well-established mouse model of cerebral malaria, namely $P$. berghei ANKA $(\mathrm{PbA})$ infection in C57BL/6 mice, have given insights that later have been confirmed by human clinical or post-mortem studies. Studies in experimental cerebral malaria of cell-cell interactions associated with small vessels in the retina have revealed the involvement of endothelial cells, astrocytes and microglia in the CNS response to the presence of intravascular PRBC. Redistribution of astrocytes is an early event following malaria inoculation in mice, and it progresses to astrogliosis and eventually to loss of astrocytes in some locations at the time when mice are exhibiting the symptoms of cerebral malaria. Microglia is activated early in the course of infection with $\mathrm{PbA}$, showing morphological and functional changes. We have suggested that activation of microglia can contribute to astrocyte damage in experimental cerebral malaria. The endothelial cells of the blood-brain barrier become compromised in their ability to exclude proteins from the brain parenchyma during the course of $\mathrm{PbA}$ infection, and also in human severe malaria, which would allow access to the brain parenchyma of factors such as circulating cytokines that otherwise might be excluded. The compromise of blood-brain barrier function in $\mathrm{PbA}$ infection is due to $\mathrm{CD} 8^{+} \mathrm{T}$ lymphocytes accumulating in the small vessels of the CNS and altering endothelial cell function via a perforin-dependent mechanism. 